\title{
Inulin and oligofructose: impact on intestinal diseases and disorders
}

\author{
Francisco Guarner* \\ Digestive System Research Unit, University Hospital Vall d'Hebron, Passeig Vall d'Hebron 119-129, Barcelona 08035, Spain
}

\begin{abstract}
A large and diverse variety of bacteria have evolved and adapted to live in the human intestinal habitat in a symbiotic arrangement that influences both physiology and pathology in the host. Symbiosis between host and flora can be optimised by prebiotics. Inulin-type fructans have been shown to improve the metabolic functions of the commensal flora. Clinical and experimental data suggest that they also improve the gut mucosal barrier. Furthermore, modulation of the trophic functions of the flora by these prebiotics could help in the prevention of inflammatory bowel diseases. The anti-inflammatory effects of inulin or oligofructose have been assessed in the rat model of distal colitis induced by dextran sodium sulphate, which histologically resembles human ulcerative colitis, and in the trinitrobenzene sulphonic acid model that resembles human Crohn's disease. Both inulin and oligofructose stimulate colonic production of SCFA and favour the growth of indigenous lactobacilli and/or bifidobacteria. These effects are associated with reduced mucosal inflammation and decreased mucosal lesion scores. Inulin has also been tested in a placebo-controlled clinical trial in patients with relapsing pouchitis. Treatment reduced endoscopic and histological parameters of inflammation of the pouch mucosa. Inulin and oligofructose may offer an opportunity to prevent chronic inflammatory intestinal disorders, and this potential should be tested in further clinical studies.
\end{abstract}

Inflammatory bowel disease: Gut flora: Animal models

The gastrointestinal tract is constitutively adapted to the analytical recognition of the external environment. The large mucosal surface is a sensitive interface that includes multiple tools and processes (receptors, secretions, sampling mechanisms) allowing a detailed scrutiny of foreign structures transiting along the tract. From a functional point of view, the gut provides the individual not only a nutritive task (digestion of food and absorption of nutrients), but also a strong line of defence against environmental aggressions. Three essential elements interacting at the mucosal surface accomplish the defensive tasks of the gut: the microflora, the mucosal barrier and the immune system (Bourlioux et al. 2003).

In recent years, special attention is being paid to the role of the commensal flora on host physiology. A large and diverse variety of bacteria have evolved and adapted to live in the human intestinal habitat. Some of these bacteria are potential pathogens and can be a source of infection and sepsis when the integrity of the mucosal barrier is physically or functionally breached. However, the interaction between host and bacteria confers important health benefits to the human host (Guarner \& Malagelada, 2003).

Evidence obtained through studies in animals bred under germfree conditions suggests that gut bacteria have important and specific functions in host homeostasis. Metabolic functions include the fermentation of non-digestible dietary substrates and endogenous mucus that results in salvage of dietary energy as SCFA, production of vitamin $\mathrm{K}$, absorption of ions $(\mathrm{Ca}, \mathrm{Mg}$, $\mathrm{Fe}$ ), etc. The diversity of genera and species within the gut microbial community provides a variety of enzymes and biochemical pathways that are distinct from the host's own constitutive resources. Protective functions of gut microflora include the barrier effect that prevents invasion by pathogens. The resident bacteria represent a crucial line of resistance to colonisation by exogenous microbes or opportunistic bacteria that are present in the gut, but their growth is restricted. The equilibrium between species of resident bacteria provides stability in the microbial population. Trophic functions of gut microflora include the control of epithelial cell proliferation and differentiation, and a crucial role in the development of the immune system. Epithelial cell differentiation is highly influenced by the interaction with resident micro-organisms as shown by the expression of a variety of genes (Hooper et al. 2001). Moreover, the cross-talk between host and bacteria at the mucosal interface plays an essential role in the development of local and systemic immunity (Fagarasan et al. 2002).

Symbiosis between host and flora can be improved by prebiotics. They are non-digestible food ingredients that beneficially affect the host by selectively stimulating growth and/or activity of one or a limited number of bacteria in the colon (Gibson \& Roberfroid, 1995). Human intestinal enzymes should not hydrolyse the prebiotic; the beneficial bacteria should selectively ferment it, and this selective fermentation should result in a beneficial effect on the health or well-being of the host (Van Loo et al. 2004).

Several studies have demonstrated that prebiotics can be used to improve the metabolic functions of the large bowel flora (see Table 1). For instance, there is ample evidence showing in human studies that inulin-type fructans improve the absorption of $\mathrm{Ca}$. In addition, animal experiments have clearly demonstrated that fermentation of oligofructose improves the hepatic metabolism of lipids and may prevent dysfunctions related with the metabolic syndrome and non-alcoholic steato-hepatitis. Other important 
Table 1. Effects of prebiotics on gut flora symbiotic functions

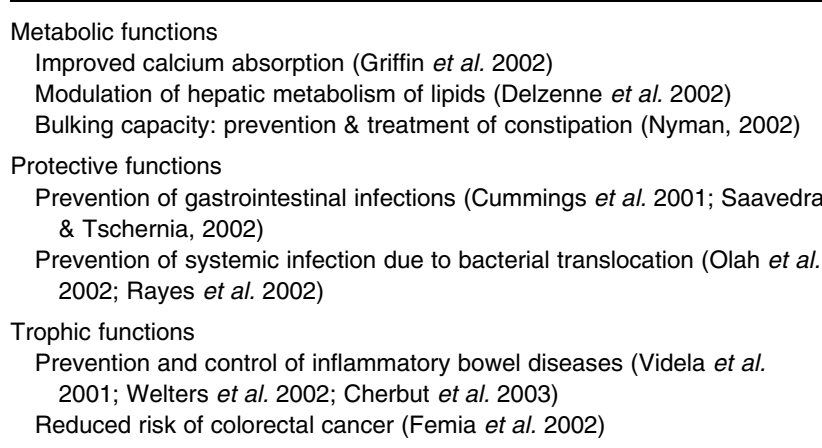

benefits of inulin-type fructans on metabolic bowel functions result from their bulking capacity and can be applied in the prevention and treatment of constipation.

Prebiotics have also been shown to improve the gut barrier (protective functions). A number of controlled clinical trials have shown that prebiotics are safe and may be effective in the prevention and treatment of gastrointestinal conditions such as community acquired diarrhoea and traveller's diarrhoea. In critical disease states, translocation of gut bacteria is associated with septic complications, and synbiotic preparations, including probiotics and prebiotics, have been used to preserve barrier function. Recent randomised controlled trials suggest that these preparations can reduce the rate of post-operative infections in liver transplant patients and the occurrence of septic complications in severe acute pancreatitis (Olah et al. 2002; Rayes et al. 2002).

Finally, modulation of the trophic functions of the flora by prebiotics is an interesting target for studies aimed at the prevention and control of inflammatory bowel diseases and the prophylaxis of colorectal cancer. The role of prebiotics in the prevention of colon cancer is reviewed in depth elsewhere in this supplement (see paper by Pool-Zobel). The purpose of the present article is to examine current evidence on the role of prebiotics in the prevention of inflammatory bowel disorders.

\section{Pathogenesis of inflammatory bowel diseases}

Crohn's disease, ulcerative colitis and pouchitis are chronic conditions of unknown aetiology characterised by persistent mucosal inflammation at different levels of the gastrointestinal tract. Evidence suggests that abnormal activation of the mucosal immune system against the enteric flora is the key event triggering inflammatory mechanisms, which induce mucosal injury and impair resolution of the lesions leading to chronic intestinal disease (Shanahan, 2001). Patients with Crohn's disease or ulcerative colitis show an increased mucosal secretion of IgG antibodies against commensal bacteria (Macpherson et al. 1996), and mucosal T-lymphocytes are hyper-reactive against antigens of the common flora, suggesting that local tolerance mechanisms are abrogated (Pirzer et al. 1991). In fact, faecal stream diversion has been shown to prevent recurrence of Crohn's disease, whereas infusion of intestinal contents to the excluded ileal segments reactivated mucosal lesions (D'Haens et al. 1998). In ulcerative colitis, short-term treatment with an enteric-coated preparation of broad-spectrum antibiotics rapidly reduced metabolic activity of the flora and mucosal inflammation (Casellas et al. 1998).
In the developed countries, there is a rising incidence of diseases due to inappropriate activation of the immune system against noninvading bacteria with antigenic potential or even auto-antigens. When the target antigens are the harmless allergens in the air, subjects are at risk of eventually developing allergies. When the target antigens are commensal bacteria living in the gut, subjects may develop inflammatory bowel diseases (Rook \& Brunet, 2002). Epidemiological evidence suggests an apparent protective effect of infections against immuno-inflammatory disorders, including Crohn's disease (Bach, 2002). Thus, the hypothesis that a restricted exposure to microbes due to excess hygiene can disturb the homeostasis of the immune system and increases susceptibility to autoimmunity is gaining credence (Rook \& Brunet, 2002).

Recent data suggest that gut bacteria populations in patients with Crohn's disease differ from that in healthy subjects (Seksisk et al. 2003). Using a molecular approach, the faecal microflora of patients showed an increase in enterobacteria (Escherichia coli group), and a substantial proportion of bacteria (30\%) not belonging to defined phylogenetic groups. In another interesting study, exhaustive investigation of the mucosal associated flora, using both conventional culture and molecular biology techniques, showed high concentrations of mucosal bacteria in patients with inflammatory bowel diseases but not in controls (Swidsinski et al. 2002). The concentrations of mucosal bacteria increased progressively with the severity of disease, and the identified bacteria were all of faecal origin.

These studies suggest that manipulation of microbial ecology in the gut by pharmacological or nutritional intervention may help in the prevention and control of inflammatory bowel disorders (Guarner, 2003). The immune response to bacteria relies on both innate and adaptive components. Innate responses in the gut are mediated by intestinal epithelial cells, which coordinate host defences by transmitting signals to underlying cells in the mucosa (Kagnoff \& Eckman, 1997). Intestinal epithelial cells express toll-like receptors that recognise conserved motifs on bacteria that are not found in higher eucaryotes. The system allows immediate recognition of potential pathogens in order to rapidly respond to an eventual challenge (Aderem \& Ulevitch, 2000). In Crohn's disease, co-culture of non-pathogenic bacteria with intestinal mucosa elicits different types of immediate cytokine response. A commensal E. coli strain stimulates the release of TNF $\alpha$ and IL- 8 by inflamed mucosa but not by non-inflamed ileal tissue (Borruel et al. 2002). Interestingly, some lactobacillus strains including Lactobacillus casei can downregulate the spontaneous release of TNF $\alpha$ by inflamed tissue, and also the inflammatory response induced by $E$. coli (Borruel et al. 2003). The effects of $L$. case $i$ on cytokine release were associated with changes in the expression of activation markers by lamina propria T-lymphocytes. An increased number of activated lymphocytes were found to undergo apoptosis (Carol et al. 2003). These findings suggest that signals generated at the mucosal surface can promote changes in the phenotype of lamina propria lymphocytes. Thus, a balanced local micro-ecology could restore immune homeostasis in Crohn's disease.

\section{Prebiotics in inflammatory bowel diseases}

The effects of probiotics and prebiotics have been tested in animal models of bowel inflammation. Mice deficient of the $I L-10$ gene spontaneously develop colitis. In the neonatal period, these knockout mice have a decreased level of Lactobacillus species in the colon and an increase in adherent and translocated bacteria 
(Madsen et al. 1999). Rectal delivery of Lactobacillus reuteri was shown to normalise counts of lactobacilli in faeces and prevented the development of colitis. In the same model, oral administration of either Lactobacillus salivarius or Bifidobacterium infantis was associated with a reduction of inflammatory activity and mucosal lesion scores (McCarthy et al. 2003). A bacterium genetically engineered to secrete the anti-inflammatory cytokine IL-10 also prevented the onset of colitis in the IL-10 knockout mouse model (Steidler et al. 2000). However, probiotic therapy with Lactobacillus plantarum did not reduce the severity of colitis induced by the chemical trinitrobenzene sulphonic acid (TNBS) in the rat (Kennedy et al. 2000). Taken together, the experimental studies with probiotics have shown that increasing the counts of lactobacilli or bifidobacteria in the colonic lumen may result in prevention of the inflammatory mucosal lesions that spontaneously develop in mice knockout of the $I L-10$ gene. Probiotic therapy, however, did not prevent mucosal injury induced by the chemical TNBS. In this model, an acute mucosal insult is induced by intracolonic administration of TNBS-ethanol. Thereafter, lesions evolve towards chronicity due to the immuno-inflammatory reaction against the hapten TNBS, and the resulting transmural lesions resemble human Crohn's disease.

The effect of the prebiotic inulin was tested in the rat model of distal colitis induced by the chemical dextran sodium sulphate (DSS), which histologically resembles human ulcerative colitis (Videla et al. 2001). Daily administration of inulin by oral route increased counts of indigenous lactobacilli in the caecal lumen and reduced the intra-colonic $\mathrm{pH}$. Interestingly, inulin feeding extended the saccharolytic region (normally right colon only) resulting in an acidic environment in the left colon. At the same time, oral inulin reduced both mucosal inflammatory activity and histological damage scores in rats with DSS-induced colitis (Fig. 1). Tissue myeloperoxidase activity, an index of neutrophil infiltration, and mucosal release of inflammatory mediators were significantly reduced in animals treated with oral inulin (Fig. 2). Furthermore, inulin-fed rats showed a reduced extent of damaged mucosa and decreased severity of crypt destruction, as compared to controls. Treatment with oral inulin was equally effective whether started prior to or during exposure to DSS.

Recent work by Moreau et al. (2003) compared the effect of oligofructose and resistant starch on mucosal inflammation

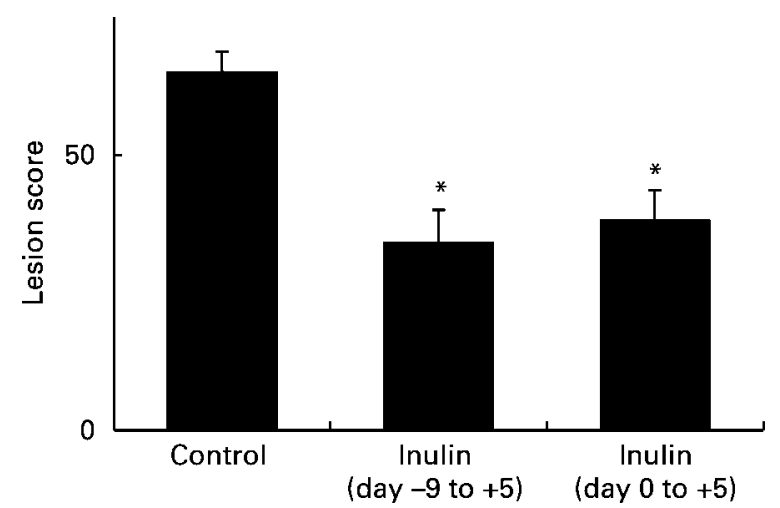

Fig. 1. Histological scores of colonic mucosal damage in rats with colitis induced by dextran sodium sulphate (DSS). Inulin-treated rats $(400 \mathrm{mg} / \mathrm{d})$ showed lower lesion scores than controls $\left({ }^{\star} P<0.05 \mathrm{v}\right.$. control). Inulin treatment started either 9 days before exposure to DSS (day -9 to +5 ) or at the same time as the exposure to DSS (day 0 to +5 ) and was continued for the 5 days on DSS.

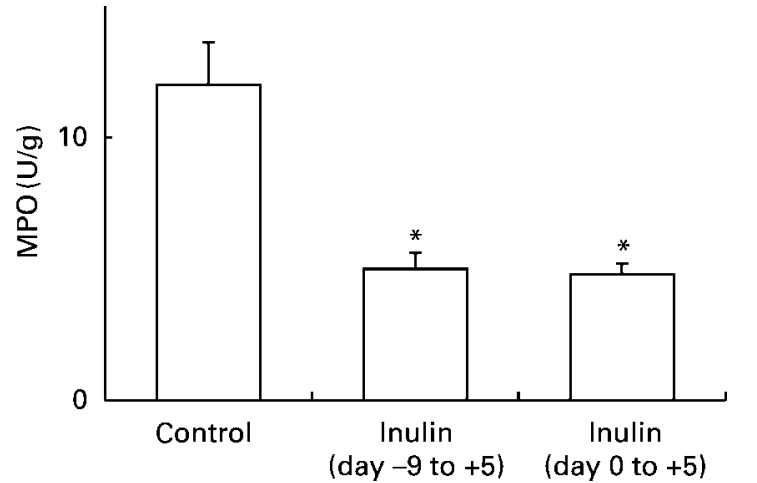

Fig. 2. Tissue myeloperoxidase (MPO) activity, as an index of neutrophil infiltration, in colonic homogenates from rats with colitis induced by dextran sodium sulphate (DSS). Inulin-treated rats $(400 \mathrm{mg} / \mathrm{d})$ showed lower tissue accumulation of MPO than controls $\left({ }^{\star} P<0.05 \mathrm{v}\right.$. control). Inulin-treatment started either 9 days before exposure to DSS (day -9 to +5 ) or at the same time as the exposure to DSS (day 0 to +5 ) and was continued for the 5 days on DSS. MPO activity in rats not subjected to DSS colitis was $0.7 \pm 0.1 \mathrm{U} / \mathrm{g}$ of tissue.

induced by DSS in the rat. Unfortunately, these authors evaluated the mucosal lesions induced by DSS using a scoring system originally designed for TNBS-induced lesions (Appleyard \& Wallace, 1995). This procedure is less appropriate since TNBS induces frank macroscopic ulcers with transmural involvement (up to the serosa) and the lesions are adjacent to areas of mucosa macro- and microscopically normal (see the original description of the model by Morris et al. 1989). Morphological features of DSS-induced colitis are different: macroscopically the colons show no ulcers, erosions or other lesions, and in light microscopy there is diffuse epithelial damage with shortening of the crypts leading to small focal erosions, and diffuse mucosal and submucosal inflammatory infiltrate but no serosal involvement (see descriptive work by Cooper et al. 1993). Lesions predominate initially in the distal and middle colon and then expand to the right colon leading to pancolitis. Surprisingly, Moreau et al. (2003) scored macroscopic lesions that were predominantly found in the caecum. In this context, the authors were able to identify the effect of resistant starch that significantly reduced lesion scores as compared with control diet. Oligofructose also reduced the scores but these changes were not significant.

The effect of oligofructose has also been tested in the TNBS model of colitis (Cherbut et al. 2003). Oral administration of oligofructose significantly reduced intra-colonic $\mathrm{pH}$, macroscopic lesion scores and tissue myeloperoxidase activity in TNBS-treated rats. In addition, oligofructose increased the concentration of lactate and butyrate as well as counts of lactic acid bacteria in caecal contents.

Inulin has also been tested in a placebo-controlled clinical trial in patients with relapsing pouchitis (Welters et al. 2002). This clinical condition is characterised by chronic mucosal inflammation of the ileal pouch-anal anastomosis in patients who had a total colectomy. The ileal pouch is surgically constructed in order to function as a faecal reservoir. The inflammatory disorder impairs the function of the reservoir and results in persistent diarrhoea with mucus and blood. Patients with mild activity were entered into the trial. Compared with placebo, 3 weeks of dietary supplementation with inulin significantly reduced endoscopic and histological parameters of inflammation of the mucosa of the ileal reservoir (Fig. 3). The effect was associated with an increase in faecal butyrate and a decrease in the counts of Bacteroides. 


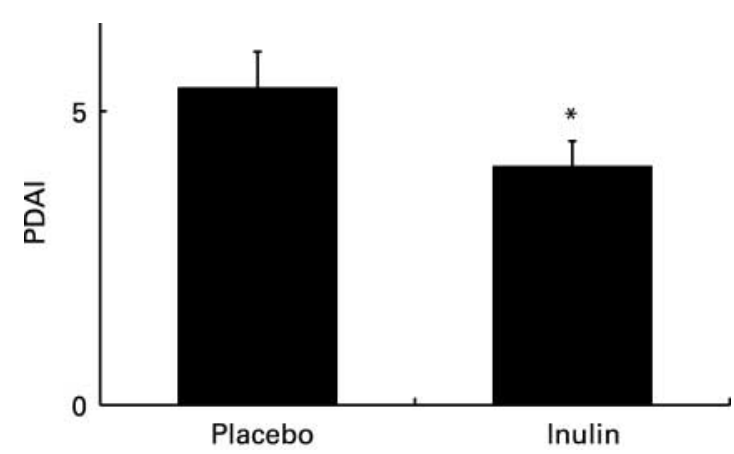

Fig. 3. Pouchitis disease activity index (PDAI) in twenty patients who received $24 \mathrm{~g}$ of inulin or placebo daily for 3 weeks in a randomised, doubleblind, cross-over study. Compared with placebo, dietary inulin supplementation reduced endoscopic and histological scores, and the total PDAI score $\left({ }^{\star} P<0.05\right.$ v. placebo).

\section{Conclusions}

In human inflammatory bowel disease, an exaggerated immune response against commensal bacteria has been demonstrated. Bacteria locally influence mucosal innate responses and cytokine signalling, and some bacteria can down-regulate mucosal inflammation. Inulin-type fructans stimulate saccharolysis in the colonic lumen and favour the growth of indigenous lactobacilli and/or bifidobacteria. These effects are associated with reduced mucosal inflammation in experimental models of inflammatory bowel disease. Inulin and oligofructose may offer an opportunity to prevent mucosal inflammatory disorders. Encouraging results have been obtained in a preliminary clinical trial, but further studies in ulcerative colitis and Crohn's disease are needed in order to confirm the applicability of inulin-type fructans for the prevention of idiopathic bowel inflammation.

\section{References}

Aderem A \& Ulevitch RJ (2000) Toll-like receptors in the induction of the innate immune response. Nature 406, 782-787.

Appleyard CB \& Wallace JL (1995) Reactivation of hapten-induced colitis and its prevention by anti-inflammatory drugs. Am J Physiol 269, G119-G125.

Bach JF (2002) The effect of infections on susceptibility to autoimmune and allergic diseases. $N$ Engl J Med 347, 911-920.

Borruel N, Carol M, Casellas F, Antolín M, de Lara F, Espín E, Naval J, Guarner F \& Malagelada JR (2002) Increased mucosal TNF $\alpha$ production in Crohn's disease can be downregulated $e x$ vivo by probiotic bacteria. Gut 51, 659-664.

Borruel N, Casellas F, Antolín M, Carol M, Llopis M, Espín E, Naval J, Guarner F \& Malagelada JR (2003) Effects of nonpathogenic bacteria on cytokine secretion by human intestinal mucosa. Am J Gastroenterol 98, 865-870.

Bourlioux P, Braesco V, Koletzko B \& Guarner F (2003) The intestine and its microflora are partners for the protection of the host. Am J Clin Nutr 78, 675-683.

Carol M, Borruel N, Antolín M, Casellas F, Guarner F \& Malagelada J-R (2003) Lactobacillus casei can overcome resistance to apoptosis in lymphocytes from patients with Crohn's disease. Gastroenterology 124, A321.

Casellas F, Borruel N, Papo M, Guarner F, Antolín M, Videla S \& Malagelada JR (1998) Antiinflammatory effects of enterically coated amoxicillin-clavulanic acid in active ulcerative colitis. Inflamm Bowel Dis 4, 1-5.
Cherbut C, Michel C \& Lecannu G (2003) The prebiotic characteristics of fructooligo-saccharides are necessary for reduction of TNBS-induced colitis in rats. J Nutr 133, 21-27.

Cooper HS, Murthy SN, Shah RS \& Sedergran DJ (1993) Clinicopathologic study of dextran sulfate sodium experimental murine colitis. Lab Invest 69, 238-249.

Cummings JH, Christie S \& Cole TJ (2001) A study of fructo oligosaccharides in the prevention of travellers' diarrhoea. Aliment Pharmacol Ther 15, 1139-1145.

D'Haens GR, Geboes K, Peeters M, Baert F, Penninckx F \& Rutgeerts P (1998) Early lesions of recurrent Crohn's disease caused by infusion of intestinal contents in excluded ileum. Gastroenterology 114, 262-267.

Delzenne NM, Daubioul C, Neyrinck A, Lasa M \& Taper HS (2002) Inulin and oligofructose modulate lipid metabolism in animals: review of biochemical events and future prospects. Br J Nutr 87, Suppl. 2, S255-S259.

Fagarasan S, Muramatsu M, Suzuki K, Nagaoka H, Hiai H \& Honjo T (2002) Critical roles of activation-induced cytidine deaminase in the homeostasis of gut flora. Science 298, 1414-1427.

Femia AP, Luceri C, Dolara P, Giannini A, Biggeri A, Salvadori M, Clune Y, Collins KJ, Paglierani M \& Caderni G (2002) Antitumorigenic activity of the prebiotic inulin enriched with oligofructose in combination with the probiotics Lactobacillus rhamnosus and Bifidobacterium lactis on azoxymethane-induced colon carcinogenesis in rats. Carcinogenesis 23, 1953-1960.

Gibson GR \& Roberfroid MB (1995) Dietary modulation of the human colonic microbiota: introducing the concept of prebiotics. J Nutr $\mathbf{1 2 5}$, $1401-1412$.

Griffin IJ, Davila PM \& Abrams SA (2002) Non-digestible oligosaccharides and calcium absorption in girls with adequate calcium intakes. $B r J$ Nutr 87, Suppl. 2, S187-S191.

Guarner F (2003) Microecology as a target for therapeutic intervention in inflammatory bowel disease. IDrugs 6, 868-873.

Guarner F \& Malagelada JR (2003) Gut flora in health and disease. Lancet 361, 512-519.

Hooper LV, Wong MH, Thelin A, Hansson L, Falk PG \& Gordon JI (2001) Molecular analysis of commensal host-microbial relationships in the intestine. Science 291, 881-884.

Kagnoff MF \& Eckmann L (1997) Epithelial cells as sensors for microbial infection. J Clin Invest 100, 6-10.

Kennedy RJ, Hoper M, Deodhar K, Kirk SJ \& Gardiner KR (2000) Probiotic therapy fails to improve gut permeability in a hapten model of colitis. Scand J Gastroenterol 35, 1266-1271.

McCarthy J, O'Mahony L, O'Callaghan L, et al. (2003) Double blind, placebo controlled trial of two probiotic strains in interleukin 10 knockout mice and mechanistic link with cytokine balance. Gut 52, 975-980.

Macpherson A, Khoo UY, Forgacs I, Philpott-Howard J \& Bjarnason I (1996) Mucosal antibodies in inflammatory bowel disease are directed against intestinal bacteria. Gut 38, 365-375.

Madsen KL, Doyle JS, Jewell LD, Tavernini MM \& Fedorak RN (1999) Lactobacillus species prevents colitis in interleukin 10 gene-deficient mice. Gastroenterology 116, 1107-1114.

Moreau NM, Martin LJ, Toquet CS, Laboisse CL, Nguyen PG, Siliart BS, Dumon HJ \& Champ MM (2003) Restoration of the integrity of rat caeco-colonic mucosa by resistant starch, but not by fructo-oligosaccharides, in dextran sulfate sodium-induced experimental colitis. $\mathrm{Br} J$ Nutr 90, 75-85.

Morris GP, Beck PL, Herridge MS, Depew WT, Szewezuk MR \& Wallace JL (1989) Hapten-induced model of chronic inflammation and ulceration in the rat colon. Gastroenterology 96, 795-803.

Nyman M (2002) Fermentation and bulking capacity of indigestible carbohydrates: the case of inulin and oligofructose. Br J Nutr 87, Suppl. 2, S163-S168.

Olah A, Belagyi T, Issekutz A, Gamal ME \& Bengmark S (2002) Randomized clinical trial of specific lactobacillus and fibre supplement to early enteral nutrition in patients with acute pancreatitis. $\mathrm{Br} J \mathrm{Surg}$ 89, $1103-1107$. 
Pirzer U, Schönhaar A, Fleischer B, Hermann E \& Meyer zum Büschenfelde KH (1991) Reactivity of infiltrating T lymphocytes with microbial antigens in Crohn's disease. Lancet 338, 1238-1239.

Rayes N, Seehofer D, Hansen S, Boucsein K, Muller AR, Serke S, Bengmark S \& Neuhaus P (2002) Early enteral supply of lactobacillus and fiber versus selective bowel decontamination: a controlled trial in liver transplant recipients. Transplantation 74, 123-127.

Rook GA \& Brunet LR (2002) Give us this day our daily germs. Biologist (London) 49, 145-149.

Saavedra JM \& Tschernia A (2002) Human studies with probiotics and prebiotics: clinical implications. Br J Nutr 87, Suppl. 2, S241-S246.

Seksik P, Rigottier-Gois L, Gramet G, Sutren M, Pochart P, Marteau P, Jian R \& Doré J (2003) Alterations of the dominant faecal bacterial groups in patients with Crohn's disease of the colon. Gut 52, 237-242.

Shanahan F (2001) Inflammatory bowel disease: immunodiagnostics, immunotherapeutics, and ecotherapeutics. Gastroenterology 120, 622-635.
Steidler L, Hans W, Schotte L, Neirynck S, Obermeier F, Falk W, Fiers W \& Remaut E (2000) Treatment of murine colitis by Lactococcus lactis secreting interleukin-10. Science 289, 1352-1355.

Swidsinski A, Ladhoff A, Pernthaler A, et al. (2002) Mucosal flora in inflammatory bowel disease. Gastroenterology 122, 44-54.

Van Loo J, Gibson GR, Probert HM, Van Loo J, Rastall RA \& Roberfroid MB (1994) Dietary modulation of the human colonic microbiota: updating the concept of prebiotics. Nutr Res Rev 17, 259-275.

Videla S, Vilaseca J, Antolín M, García-Lafuente A, Guarner F, Crespo E, Casalots J, Salas A \& Malagelada JR (2001) Dietary inulin improves distal colitis induced by dextran sodium sulfate in the rat. Am J Gastroenterol 96, 1486-1493.

Welters CF, Heineman E, Thunnissen FB, van den Bogaard AE, Soeters PB \& Baeten CG (2002) Effect of dietary inulin supplementation on inflammation of pouch mucosa in patients with an ileal pouch-anal anastomosis. Dis Colon Rectum 45, 621-627. 